\title{
Wiener and Hyper-Wiener Indices of Polygonal Cylinder and Torus
}

\author{
Zhi-Ba Peng, ${ }^{1}$ Abdul Rauf Nizami, ${ }^{2}$ Zaffar Iqbal, ${ }^{3}$ Muhammad Mobeen Munir ${ }^{(D},{ }^{4}$ \\ Hafiz Muhammad Waqar Ahmed, ${ }^{5}$ and Jia-Bao Liu $\mathbb{D D}^{1}$
}

${ }^{1}$ School of Mathematics and Physics, Anhui Jianzhu University, Hefei 230601, China

${ }^{2}$ Department of Mathematics, University of Central Punjab, Lahore, Pakistan

${ }^{3}$ Department of Mathematics, University of Gujrat, Gujrat, Pakistan

${ }^{4}$ Department of Mathematics, University of the Punjab, Lahore, Pakistan

${ }^{5}$ Department of Mathematics, GC University, Lahore, Pakistan

Correspondence should be addressed to Muhammad Mobeen Munir; mmunir.math@pu.edu.pk

Received 23 August 2020; Revised 17 January 2021; Accepted 8 February 2021; Published 9 April 2021

Academic Editor: M. Irfan Uddin

Copyright ( $\odot 2021$ Zhi-Ba Peng et al. This is an open access article distributed under the Creative Commons Attribution License, which permits unrestricted use, distribution, and reproduction in any medium, provided the original work is properly cited.

In this study, we first introduce polygonal cylinder and torus using Cartesian products and topologically identifications and then find their Wiener and hyper-Wiener indices using a quick, interesting technique of counting. Our suggested mathematical structures could be of potential interests in representation of computer networks and enhancing lattice hardware security.

\section{Introduction}

The Wiener index was introduced by Harold Wiener in 1947 as the sum of distances between all pairs of vertices in the molecular graph of an alkane, with the evident aim to provide a measure of the compactness of the respective hydrocarbon molecule [1].

Wiener index has remarkable variety of chemical applications. Wiener himself used it to predict boiling points, molar volumes, refractive indices, and heats of vaporization of alkanes [2-5]. Gutman showed in [6] that the Wiener index measures the area of the surface of the respective molecule and thus reflects its compactness. As a consequence, it is related to intermolecular forces, especially in the case of hydrocarbons where polar groups are absent [7-9]. Wagner proved that every integer greater or equal to 470 is the Wiener index for a class of trees that is connected to partitions of integers [10]. Wand and $\mathrm{Yu}$ proved that every integer $n>10^{8}$ is the Wiener index of some short caterpillar tree with at most six nonleaf vertices [11]. Hua and Wang gave a new sufficient condition for a connected graph to be traceable by means of Harary index [12]. Hua and Ning gave some tight sufficient conditions for traceability and Hamiltonicity of connected graphs with given minimum degree, in terms of Wiener and Harary indices [13]. Recently, Khalid and Idrees computed the Wiener index of Dutch windmill graph [14], and Thilaga and Sarasija found Wiener index of unitary addition Cayley graphs [15].

Physical and chemical properties of organic substances, which can be expected to depend on the area of the molecular surface and/or on the branching of the molecular carbon-atom skeleton, are usually well correlated with the Wiener index. Among them are the heats of formation, vaporization and atomization, density, boiling point, critical pressure, refractive index, surface tension and viscosity of various, acyclic and cyclic, saturated and unsaturated as well as aromatic hydrocarbon species, velocity of ultra sound in alkanes and alcohols, rate of electroreduction of chlorobenzenes, etc. [16]. Of particular practical importance is the prediction of the behaviour of organic substances in gas chromatography. For instance, chromatographic retention times of monoalkyl- and 0-dialkylbenzenes can be modeled by the Wiener index [17]. 
Since the pharmacological activity of a substance is related to some of its physico-chemical properties, it is not surprising that attempts have been made to use the Wiener index in designing new drugs [18-20] and hence has a strong impact on stock market. The Wiener index is correlated to cytostatic and antihistaminic activities of certain pharmacologically interesting compounds as well as to their estronbinding affinities [20]. It is also used to study $n$-octanol to water partition coefficient, a physico-chemical parameter of profound importance, for the forecasting of pharmacological activity of many compounds. To learn more about Wiener index, see [21-33].

The hyper-Wiener index was introduced by Randic in 1993 [34]. It is also used to predict physico-chemical properties of organic compounds, particularly to pharmacology, agriculture, and environment protection [35]. In 2008, Khalifeh et al. gave interesting results about the hyperWiener index of graph operations [36]. Recently, Raghisa and Nazeran computed the hyper-Wiener index of Dutch windmill graph [14], and Thilaga and Sarasija found the hyper-Wiener index of unitary addition Cayley graphs [15]. For details, see [9, 26, 37-40].

This paper is concerned with the polygonal cylinder and torus and their topological invariants, Wiener and hyperWiener indices. First of all, the way we are introducing the polygonal cylinder and torus is unique and useful. Although one can see such a cylinder and torus in literature, precise definitions do not exist. We give precise topological definitions so that one can clearly understand the constructions. Our cylinder depends on two parameters, $m$ and $n$, which determine its base and height and hence help further study about it. Similarly, torus is obtained after identifying the ends of the cylinder. Secondly, we computed the indices directly by definition, not by the usual way of recovering them from the Hosoya polynomial. Although the Wiener and hyper-Wiener indices have profound importance because of their strong correlation to various chemical and pharmaceutical properties of chemical compounds, our purpose of computing these indices is the classification of connected graphs. Finally, we hope the cylinder and torus will play a useful role in studying those graphs which contain them as subgraphs. The lattice sheet of the cylinder can be used in enhancing security in semiconductors.

\section{Preliminary Notes}

A graph $G$ is a pair $(V, E)$, where $V$ is the set of vertices and $E$ the set of edges. The edge $e$ between two vertices $u$ and $v$ is denoted by $(u, v)$. A path from a vertex $v$ to a vertex $w$ is a sequence of vertices and edges that starts from $v$ and stops at $w$. The number of edges in a path is the length of that path. The distance between two vertices $u$ and $v$, denoted by $d(u, v)$, is the length of the shortest path between them. The diameter of $G$, denoted by $d(G)$, is the longest distance in $G$. A graph is said to be connected if there is a path between any two of its vertices.

Definition 1. A function $I$ which assigns to every connected graph $G$ a unique number $I(G)$ is called a graph invariant. Instead of the function $I$, it is custom to say the number $I(G)$ as the invariant. An invariant of a molecular graph which can be used to determine structure-property or structureactivity correlation is called the topological index. A topological index is said to be distance-based if it depends on distances of the vertices of the graph.

In the following, we shall mean by $d(v, G)$ the sum of distances of $v$ with all vertices of a connected graph $G$, and we shall mean by $d(G, k)$ the number of pairs of vertices of $G$ laying at distance $k$ from each other.

Definition 2 (see $[1,41]$ ). The Wiener index $W(G)$ of a graph $G$ is defined as

$$
W(G)=\sum_{i<j} d\left(v_{i}, v_{j}\right)=\frac{1}{2} \sum_{v \in V} d(v, G)=\frac{1}{2} \sum_{k \geq 1} k d(G, k) .
$$

Definition 3 (see $[34,41]$ ). The hyper-Wiener index WW $(G)$ of a graph $G$ is defined as

$$
\mathrm{WW}(G)=\frac{1}{2} \sum_{i<j}\left[d\left(v_{i}, v_{j}\right)^{2}+d\left(v_{i}, v_{j}\right)\right]=\frac{1}{2} \sum_{k \geq 1}\left(k^{2}+k\right) d(G, k) \text {. }
$$

\section{Main Results}

\subsection{Polygonal Cylinder}

Definition 4. Consider the Cartesian product $P_{m} \times P_{n}$ of paths $P_{m}, m \geq 4$, and $P_{n}, n \geq 2$, with vertices $u_{1}, u_{2}, \ldots, u_{m}$ and $v_{1}, v_{2}, \ldots, v_{n}$, respectively. Identify the vertices $\left(u_{1}, v_{1}\right),\left(u_{1}, v_{2}\right), \ldots,\left(u_{1}, v_{n}\right)$ with the vertices $\left(u_{m}, v_{1}\right)\left(u_{m}, v_{2}\right), \ldots,\left(u_{m}, v_{n}\right)$, respectively, and identify the edge $\left(\left(u_{1}, v_{i}\right),\left(u_{1}, v_{i+1}\right)\right)$ with the edge $\left(\left(u_{m}, v_{i}\right),\left(u_{m}, v_{i+1}\right)\right)$, where $1 \leq i \leq n-1$. What we receive is the polygonal (actually, $C_{m, n}$; we may call it $(m-1)$-gonal cylinder. You can see $P_{5} \times P_{4}$ in Figure 1 .

For brevity, we shall use the symbol $w_{i, j}\left(v_{i j}\right.$ or simply $\left.i j\right)$ to represent the vertex $\left(u_{i}, v_{j}\right)$ of $C_{m, n}$. In Figure 2, you can see the grid form of $C_{5,4}$ along with simple labels.

$\mathrm{P}_{5} \times \mathrm{P}_{4}$ is shown in Figure 3 . 

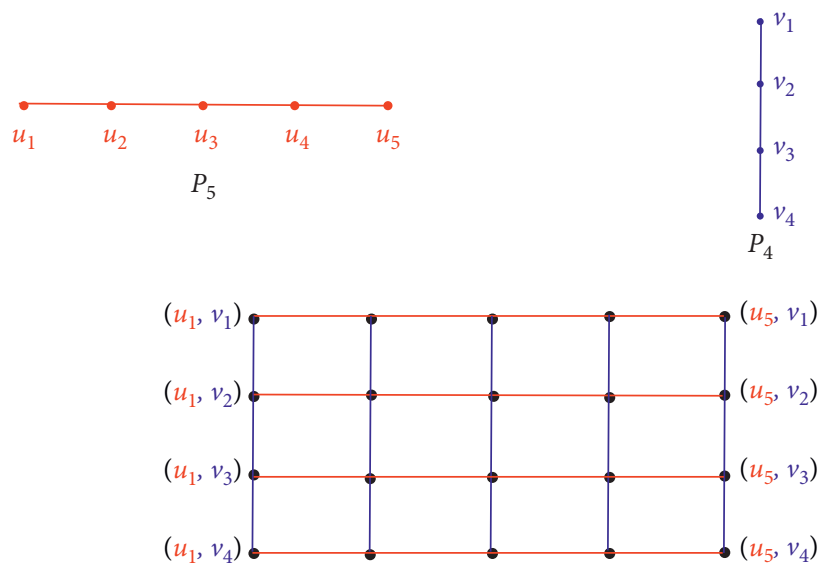

Figure 1: $P_{5} \times P_{4}$.

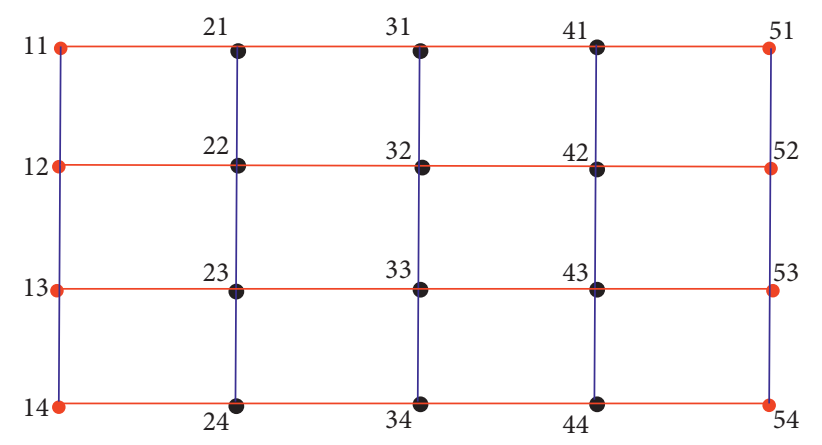

Figure 2: $C_{5,4}$.

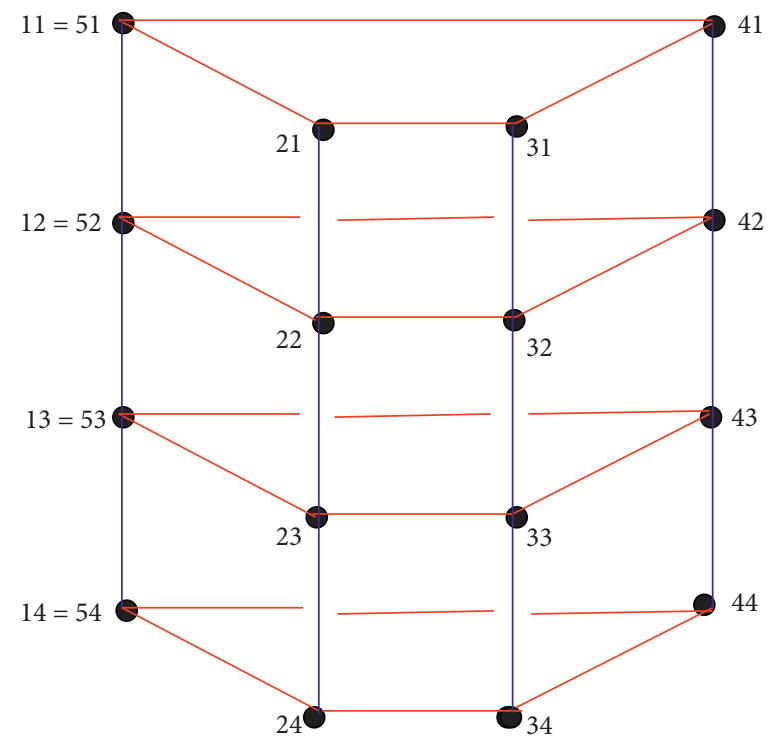

FIgURE 3: $\mathrm{C}_{5,4}$. 
In all the following results, $n \geq 3$.

A notational digression: by \# $\left(A_{i}\right)$, we shall mean the number of times $A_{i}$ appears in $D$, and by \# $(a) \in A_{i}$, we shall mean the number of times $a$ appears in $A_{i}$.

To avoid repetitions, only the necessary parts of the proofs are given.

Theorem 1. Let $m$ be odd. Then, the Wiener and hyperWiener indices of the polygonal cylinder $C_{m, n}$ are

(1) $W\left(C_{m, n}\right)=(n / 24)(m-1)^{2}\left[4 n^{2}+(3 m-3) n-4\right]$

(2) $W W\left(C_{m, n}\right)=(n / 48)(m-1)^{2}\left[n m^{2}+\left(2 n^{2}+n-2\right)\right.$ $\left.m+\left(2 n^{3}+2 n^{2}-2 n-2\right)\right]$

Proof. In order to count all distinct paths, we need to find distance of each vertex with every other vertex of $C_{m, n}$. For convenience, we shall write lengths of paths in the form of a matrix $D$, which we shall call the distance matrix. $D$ corresponding to the polygonal cylinder $C_{m, n}$ is symmetric of order $(m-1) n \times(m-1) n$. Each row of $D$ represents the distances from a vertex $v_{i j}$ to the vertices $v_{1,1}, v_{1,2}, \ldots, v_{1, n}, v_{2,1}, v_{2,2}, \ldots, v_{2, n}, \ldots, v_{m-1,1}, v_{m-1,2}, \ldots, v_{m}$ $-1, n$, respectively. Since we need distinct paths, we shall consider only its upper-triangular part. For this, we represent the upper-triangular part by submatrices. There are $(m+1 / 2)$ distinct submatrices $A_{0}, A_{1}, A_{2}, A_{3}, \ldots, A_{(m-3 / 2)}$, and $A_{(m-1 / 2)}$. All these submatrices are symmetric, each having order $n \times n$. Each $A_{i}$ appears $m-1$ times except $A_{(m-1 / 2)}$, which appears $(m-1 / 2)$ times. $A_{0}$ appears only on the main diagonal of $D . A_{i}, 1 \leq i \leq(m-3 / 2)$, appears $m-$ $(i+1)$ times in $i$ th secondary diagonal and $i$ times in $(m-$ $(i-1))$ th secondary diagonal. $A_{(m-1 / 2)}$ appears only in $(m-1 / 2)$ th secondary diagonal. Thus, the rows of the distance matrix $D$ are

$$
\begin{aligned}
R_{1} & =\left(A_{0}, A_{1}, A_{2}, \ldots, A_{(m-3 / 2)}, A_{(m-1 / 2)}, A_{(m-3 / 2)}, A_{(m-5 / 2)}, \ldots, A_{2}, A_{1}\right), \\
R_{2} & =\left(A_{1}, A_{0}, A_{1}, \ldots, A_{(m-5 / 2)}, A_{(m-3 / 2)}, A_{(m-1 / 2)}, A_{(m-3 / 2)}, \ldots, A_{3}, A_{2}\right), \\
R_{3} & =\left(A_{2}, A_{1}, A_{0}, \ldots, A_{(m-7 / 2)}, A_{(m-5 / 2)}, A_{(m-3 / 2)}, A_{(m-1 / 2)}, \ldots, A_{4}, A_{3}\right), \\
& \vdots \\
R_{(m-1) n} & =\left(A_{1}, A_{2}, A_{3}, \ldots, A_{(m-5 / 2)}, A_{(m-3 / 2)}, A_{(m-1 / 2)}, A_{(m-3 / 2)}, \ldots, A_{0}\right) .
\end{aligned}
$$

Now, we give the entries of the submatrices. Since $A_{0}$ lies on the main diagonal of $D$, only its upper-triangular part contributes towards counting paths and is

$$
A_{0}=\left(\begin{array}{ccccccc}
0 & 1 & 2 & 3 & \cdots & n-2 & n-1 \\
& 0 & 1 & 2 & \cdots & n-3 & n-2 \\
& & 0 & 1 & \cdots & n-4 & n-3 \\
& & & \ddots & \vdots & \vdots & \vdots \\
& & & & 0 & 1 & 2 \\
& & & & & 0 & 1 \\
& & & & & & \\
& & & & & & 0
\end{array}\right) .
$$

However, all the entries of $A_{i}, 1 \leq i \leq(m-1 / 2)$, contribute towards counting. Since each $A_{i}$ is symmetric, we give only its upper-triangular part:

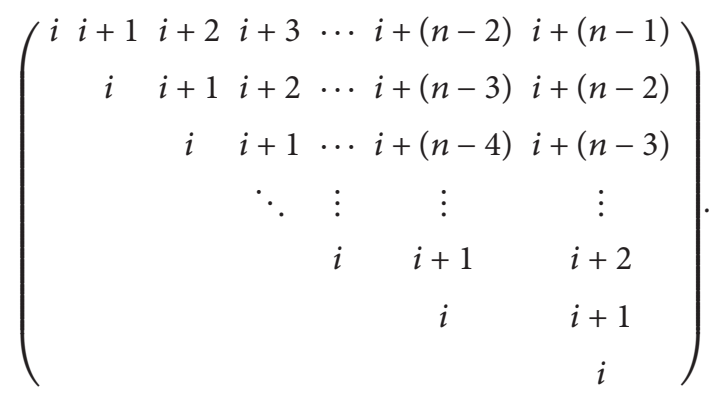

Now, we count the number of paths in $C_{m, n}$. There are $(m-1 / 2)+n-1$ distinct paths; we shall denote the number of paths of length $i$ by $c_{i}, 1 \leq i \leq(m-1 / 2)+n-1$. Depending on lengths, the paths can be divided into four groups: $\quad c_{k}, 1 \leq k \leq n-1, \quad c_{n+k}, 0 \leq k \leq(m-1 / 2)-$ $n-1, c_{(m-1 / 2)}$, and $c_{(m-1 / 2)+k}, 1 \leq k \leq n-1$ : 


$$
\begin{aligned}
& c_{k}=\left[\#(k) \in A_{0}\right] \times\left[\#\left(A_{0}\right)\right]+\left[\#(k) \in A_{1}\right] \times\left[\#\left(A_{1}\right)\right] \\
& +\left[\#(k) \in A_{2}\right] \times\left[\#\left(A_{2}\right)\right]+\cdots+\left[\#(k) \in A_{k-1}\right] \times\left[\#\left(A_{k-1}\right)\right]+\left[\#(k) \in A_{k}\right] \times\left[\#\left(A_{k}\right)\right] \\
& =(n-k)(m-1)+2(n-(k-1))(m-1)+2(n-(k-2))(m-1)+\cdots+2(n-1)(m-1)+n(m-1) \\
& =(m-1)(n-k+2(n-(k-1)+2(n-(k-2))+2(n-(k-3))+\cdots+2(n-1)+n) \\
& =(m-1)(n-k+2 n-2(k-1)+2 n-2(k-2)+2 n-2(k-3)+\cdots+2 n-2(1)+n) \\
& \cdot(m-1)(2 k n-2(1+2+3+\cdots+k-1)-k) \\
& =(m-1)(2 k n-(k-1)(k)-k)=(m-1)\left(2 k n-k^{2}\right), \\
& c_{(m-1 / 2)}=\left[\#\left(\frac{m-1}{2}\right) \in A_{0}\right] \times\left[\#\left(A_{0}\right)\right]+\left[\#\left(\frac{m-1}{2}\right) \in A_{1}\right] \times\left[\#\left(A_{1}\right)\right] \\
& +\left[\#\left(\frac{m-1}{2}\right) \in A_{2}\right] \times\left[\#\left(A_{2}\right)\right]+\left[\#\left(\frac{m-1}{2}\right) \in A_{3}\right] \times\left[\#\left(A_{3}\right)\right]+\cdots+\left[\#\left(\frac{m-1}{2}\right) \in A_{(m-1 / 2)-1}\right] \times\left[\#\left(A_{(m-1 / 2)-1}\right)\right] \\
& +\left[\#\left(\frac{m-1}{2}\right) \in A_{(m-1 / 2)}\right] \times\left[\#\left(A_{(m-1 / 2)}\right)\right] \\
& =\left(n-\frac{m-1}{2}\right)(m-1)+2\left(n-\frac{m-1}{2}+1\right)(m-1)+\left(n-\frac{m-1}{2}+2\right)(m-1) \\
& +\left(n-\frac{m-1}{2}+3\right)(m-1)+\cdots+\left(n-\frac{m-1}{2}+\frac{m-1}{2}-1\right)(m-1)+n\left(\frac{m-1}{2}\right) \\
& =\left(n-\frac{m-1}{2}\right)+2\left[\left(\frac{1}{2} \frac{m-3}{2}\right)\left(2 n-\frac{m-1}{2}\right)\right](m-1)+\frac{n}{2}(m-1) \\
& =\left[\left(\frac{1}{2}(3 n-m+1)+(m-3)\left(n-\frac{m-1}{4}\right)\right)\right](m-1) \\
& =\frac{-1}{4}(m-1)\left[m^{2}-(4 n+2) m+(6 n+1)\right] .
\end{aligned}
$$

Now, $c_{(m-1 / 2)+k}, 1 \leq k \leq n-1-(m-1 / 2)$ :

$$
\begin{aligned}
\mathcal{c}_{(m-1 / 2)+k}= & {\left[\#\left(\frac{m-1}{2}+k\right) \in A_{0}\right] \times\left[\#\left(A_{0}\right)\right]+\left[\#\left(\frac{m-1}{2}+k\right) \in A_{1}\right] \times\left[\#\left(A_{1}\right)\right] } \\
& +\left[\#\left(\frac{m-1}{2}+k\right) \in A_{2}\right] \times\left[\#\left(A_{2}\right)\right]+\left[\#\left(\frac{m-1}{2}+k\right) \in A_{3}\right] \\
& \times\left[\#\left(A_{3}\right)\right]+\cdots+\left[\#\left(\frac{m-1}{2}+k\right) \in A_{(m-1 / 2)-1}\right] \times\left[\#\left(A_{(m-1 / 2)}\right)\right]+A_{(m-1 / 2)} \\
= & \left(n-\frac{m-1}{2}-k\right)(m-1)+2\left(n-\frac{m-1}{2}+1-k\right)(m-1)+\left(n-\frac{m-1}{2}+2-k\right)(m-1) \\
& +\left(n-\frac{m-1}{2}+3-k\right)(m-1)+\cdots+\left(n-\frac{m-1}{2}+\frac{m-1}{2}-k-1\right)(m-1)+2(n-k)\left(\frac{m-1}{2}\right) \\
= & \frac{-1}{4}(m-1)\left[m^{2}-2(2 n-2 k+1) m+(4 n-4 k+1)\right] .
\end{aligned}
$$


Finally, $c_{n+k}, 0 \leq k \leq(m-1 / 2)-1$ :

$$
\begin{aligned}
c_{n+k}= & {\left[\#(n+k) \in A_{k+1}\right] \times\left[\#\left(A_{k+1}\right)\right]+\left[\#(n+k) \in A_{k+2}\right] \times\left[\#\left(A_{k+2}\right)\right] } \\
& +\left[\#(n+k) \in A_{k+3}\right] \times\left[\#\left(A_{k+3}\right)\right]+\cdots+\left[\#(n+k) \in A_{(m-1 / 2)-1}\right] \times\left[\#\left(A_{(m-1 / 2)-1}\right)\right] \\
& +\left[\#(n+k) \in A_{(m-1 / 2)}\right] \times\left[\#\left(A_{(m-1 / 2)}\right)\right] \\
= & 2(m-1)+2(2)(m-1)+2(3)(m-1)+\cdots+2\left(\frac{m-1}{2}-k-1\right)(m-1) \\
& +2\left(\frac{m-1}{2}-k\right)\left(\frac{m-1}{2}\right)=\left(\frac{m-1}{2}-k\right)^{2} .
\end{aligned}
$$

$1 W\left(C_{m, n}\right)=\sum_{k=1}^{(m-1 / 2)-1}[k] c_{k}+[(m-1 / 2)] c_{(m-1 / 2)}+$ $\sum_{k=1}^{n-1-(m-1 / 2)}[(m-1 / 2)+k] c_{(m-1 / 2)+k}+\sum_{k=0}^{(m-1 / 2)-1}[n+$ $k] c_{n+k}=\sum_{k=1}^{(m-1 / 2)-1}[k](m-1)\left(2 n k-k^{2}\right)+[(m-1 /$ 2)] $(-1 / 4)(m-1)\left[m^{2}-(4 n+2) m+(6 n+1)\right]+$ $\sum_{k=1}^{n-1-(m-1 / 2)}[(m-1 / 2)+k] \quad(-1 / 4)(m-1)\left[m^{2}-2\right.$ $(2 n-2 k+1) m+(4 n-4 k+1)]+\sum_{k=0}^{(m-1 / 2)-1}[n+k]$ $(1 / 4)(m-1)[m-2 k-1]^{2}=\left[(-1 / 192)(m-1)^{2}(m-\right.$ 3)) $\left.\left(3 m^{2}-16 m n-12 m+32 n+9\right)\right]+\left[(-1 / 8)(m-1)^{2}\right.$ $\left.\left(m^{2}-4 m n-2 m+6 n+1\right)\right]+\left[(1 / 96)(m-1)^{2}(-2 n+\right.$ $\left.1+m)\left(m^{2}-10 m n-8 n^{2}+2 m+2 n-3\right)\right]+[(1 / 96)$ $\left.\left.(m-1)^{2}(m+1)\right)\left(m^{2}+8 m n-4 m+3\right)\right]=(n / 24)$ $(m-1)^{2}\left[4 n^{2}+(3 m-3) n-4\right]$.

$2 \mathrm{WW}\left(C_{m, n}\right)=(1 / 2) \sum_{k=1}^{(m-1 / 2)-1}\left[k^{2}+k\right] c_{k}+\quad(1 / 2)[(m-$ $\left.1 / 2)^{2}+(m-1 / 2)\right] c_{(m-1 / 2)}+(1 / 2) \sum_{k=1}^{n-1-(m-1 / 2)}[((m-1 /$ $\left.2)+k)^{2}+((m-1 / 2)+k)\right] \quad c_{(m-1 / 2)+k}+(1 / 2) \quad \sum_{k=0}^{(m-1 / 2)-1}$ $\left[(n+k)^{2}+(n+k)\right] c_{n+k}=(1 / 2) \sum_{k=1}^{(m-1 / 2)-1}\left[k^{2}+k\right](m-$ 1) $\left(2 n k-k^{2}\right)+(1 / 2)\left[(m-1 / 2)^{2}+(m-1 / 2)\right] \quad(-1 / 4)$ $(m-1)\left[m^{2}-(4 n+2) m+(6 n+1)\right]+(1 / 2) \sum_{k=1}^{n-1-(m-1 / 2)}$ $\left[((m-1 / 2)+k)^{2}+((m-1 / 2)+k)\right] \quad(-1 / 4)(m-1)$ $\left[m^{2}-2(2 n-2 k+1) m+(4 n-4 k+1)\right]+(1 / 2) \sum_{k=0}^{(m-1 / 2)-1}$ $\left[(n+k)^{2}+(n+k)\right](1 / 4)(m-1)[m-2 k-1]^{2}=[(-1 /$ 1920) $\left.(m-1)^{2}(m+1)\right)(m-3)\left(6 m^{2}-30 m n-27 m+\right.$ $70 n+25)]+\left[(-1 / 32)(m-1)^{2}(m+1)\left(m^{2}-4 m n-2 m+\right.\right.$ $6 n+1)]+\left[(1 / 384)(m-1)^{2}(-2 n+1+m)\left(m^{3}-6 m^{2} n-\right.\right.$ $\left.\left.12 m n^{2}-8 n^{3}+3 m^{2}-12 m n-12 n^{2}-m+2 n-3\right)\right]+[(1 /$ 1920) $\quad(m-1)^{2}(m+1) \quad\left(m^{3}+10 m^{2} n+40 m n^{2}-9 m+\right.$
$30 n)]=(n / 48)(m-1)^{2}\left(m^{2} n+2 m n^{2}+2 n^{3}+m n+2 n^{2}-\right.$ $2 m-2 n-2)$.

Example 1. For $n=5$ and $m=5$, the distance matrix of order $20 \times 20$ is $D=\left(\begin{array}{cccc}A_{0} & A_{1} & A_{2} & A_{1} \\ A_{1} & A_{0} & A_{1} & A_{2} \\ A_{2} & A_{1} & A_{0} & A_{1} \\ A_{1} & A_{2} & A_{1} & A_{0}\end{array}\right)$, where the submatrices are $A_{0}=\left(\begin{array}{lllll}0 & 1 & 2 & 3 & 4 \\ 1 & 0 & 1 & 2 & 3 \\ 2 & 1 & 0 & 1 & 2 \\ 3 & 2 & 1 & 0 & 1 \\ 4 & 3 & 2 & 1 & 0\end{array}\right), A_{1}=\left(\begin{array}{lllll}1 & 2 & 3 & 4 & 5 \\ 2 & 1 & 2 & 3 & 4 \\ 3 & 2 & 1 & 2 & 3 \\ 4 & 3 & 2 & 1 & 2 \\ 5 & 4 & 3 & 2 & 1\end{array}\right)$, and $A_{2}=\left(\begin{array}{lllll}2 & 3 & 4 & 5 & 6 \\ 3 & 2 & 3 & 4 & 5 \\ 4 & 3 & 2 & 3 & 4 \\ 5 & 4 & 3 & 2 & 3 \\ 6 & 5 & 4 & 3 & 2\end{array}\right)$. The Wiener and hyper-Wiener indices for odd $m<2 n-1$ and for $n=5$ are, respectively, $W=2(m-1)^{2}(m+4) \quad$ and $\mathrm{WW}=(1 / 6)(m-1)^{2}\left(2 m^{2}+17 m+75\right)$. The graphs of $W$ and WW are shown in Figure 4.

Theorem 2. For even $m$, we have

(1) $W\left(C_{m, n}\right)=(n / 24)(m-1)\left[3 n m^{2}+\left(4 n^{2}-6 n-4\right)\right.$ $\left.m-4 n^{2}+4\right]$

(2) $W W\left(C_{m, n}\right)=(n / 48)(m-1)\left[n m^{3}+2\left(n^{2}-1\right) m^{2}+2\right.$ $\left.\left(n^{3}-3 n\right) m-2 n^{3}-4 n^{2}+2 n+4\right]$

Proof. The rows of the distance matrix $D$ are 


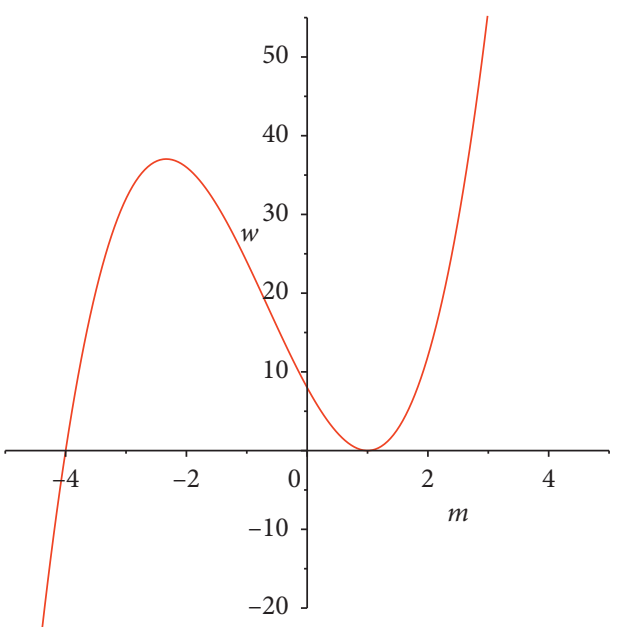

(a)

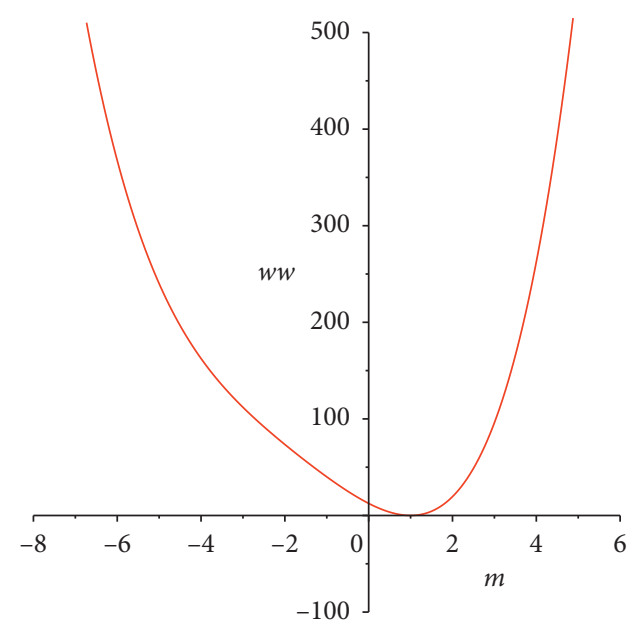

(b)

Figure 4: (a) Wiener index and (b) hyper-Wiener index for $n=5$.

$$
\begin{aligned}
R_{1} & =\left(A_{0}, A_{1}, A_{2}, \ldots, A_{(m / 2)-2}, A_{(m / 2)-1}, A_{(m / 2)-1}, A_{(m / 2)-2}, A_{(m / 2)-3}, \ldots, A_{2}, A_{1}\right), \\
R_{2} & =\left(A_{1}, A_{0}, A_{1}, \ldots, A_{(m / 2)-3}, A_{(m / 2)-2}, A_{(m / 2)-1}, A_{(m / 2)-1}, A_{(m / 2)-2}, \ldots, A_{3}, A_{2}\right), \\
R_{3} & =\left(A_{2}, A_{1}, A_{0}, \ldots, A_{(m / 2)-4}, A_{(m / 2)-3}, A_{(m / 2)-2}, A_{(m / 2)-1}, A_{(m / 2)-1}, \ldots, A_{4}, A_{3}\right), \\
& \vdots \\
R_{(m-1) n} & =\left(A_{1}, A_{2}, \ldots, A_{(m / 2)-3}, A_{(m / 2)-2}, A_{(m / 2)-1}, A_{(m / 2)-2}, \ldots, A_{2}, A_{1}, A_{0}\right) .
\end{aligned}
$$

Each submatrix $A_{0}, A_{1}, A_{2}, A_{3}, \ldots, A_{(m / 2)-2}$ and $A_{(m / 2)-1}$ appears $m-1$ times. $A_{0}$ appears only on the main diagonal of $D . A_{i}, 1 \leq i \leq(m / 2)-1$, appears $m-(i+1)$ times in $i$ th secondary diagonal and $i$ times in $[m-(i-1)]$ th secondary diagonal. These submatrices are the same as in Theorem 1 . The paths are divided into three groups: $c_{k}, 1 \leq k \leq n-1$, $c_{k+n}, 0 \leq k \leq(m / 2)-n-1$, and $c_{(m / 2)+k}, 0 \leq k \leq n-2 . c_{k}$ and $c_{k+n}$ are already given in Theorem 1 . We need only $c_{(m / 2)+k}$ :

$$
\begin{aligned}
c_{(m / 2)+k}= & \left.\#\left(\frac{m}{2}+k\right) \in A_{(m / 2)-n+(k+1)}\right] \times\left[\#\left(A_{(m / 2)-n+(k+1)}\right)\right]+\left[\#\left(\frac{m}{2}+k\right) \in A_{(m / 2)-n+(k+2)}\right] \times\left[\#\left(A_{(m / 2)-n+(k+2)}\right)\right] \\
& +\left[\#\left(\frac{m}{2}+k\right) \in A_{(m / 2)-n+(k+3)}\right] \times\left[\#\left(A_{(m / 2)-n+(k+3)}\right)\right]+\cdots+\left[\#\left(\frac{m}{2}+k\right) \in A_{(m / 2)-2}\right] \times\left[\#\left(A_{(m / 2)-2}\right)\right] \\
& +\left[\#\left(\frac{m}{2}+k\right) \in A_{(m / 2)-1}\right] \times\left[\#\left(A_{(m / 2)-1}\right)\right] \\
= & 2(m-1)+2(2)(m-1)+2(3)(m-1)+\cdots+2(n-(k+2))(m-1)+2(n-(k+1))(m-1) \\
= & (m-1)[2(1+2+3+\cdots+(n-(k+2))+(n-(k+1))] \\
= & (m-1)[(n-(k+1))(n-k)]=(m-1)\left[(n-k)^{2}-(n-k)\right] .
\end{aligned}
$$

\subsection{Polygonal Torus}

Definition 5. Consider the Cartesian product $P_{m} \times P_{n}$ of paths $P_{m}, m \geq 4$, and $P_{n}, n \geq 4$, with vertices $u_{1}, u_{2}, \ldots, u_{m}$ and $v_{1}, v_{2}, \ldots, v_{n}$, respectively. Then, identify, respectively, the vertices $\left(u_{1}, v_{j}\right)$ with the vertices $\left(u_{m}, v_{j}\right)$ for $1 \leq j \leq n$ and identify, respectively, the edges $\left(\left(u_{1}, v_{i}\right),\left(u_{1}, v_{i+1}\right)\right)$, with the edges $\left(\left(u_{m}, v_{i}\right),\left(u_{m}, v_{i+1}\right)\right)$ for $1 \leq i \leq n-1$; this way we receive a polygonal cylinder. Finally, identify, respectively, the vertices $\left(u_{i}, v_{1}\right)$ with the vertices $\left(u_{i}, v_{m}\right)$ for $1 \leq i \leq m$ and identify, respectively, the edges $\left(\left(u_{i}, v_{1}\right),\left(u_{i+1}, v_{1}\right)\right)$ with the edges $\left(\left(u_{i}, v_{n}\right),\left(u_{i+1}, v_{n}\right)\right)$ for $1 \leq i \leq m$. What we receive is the polygonal torus $T_{m, n}$. For instance, you can see the construction of $T_{4,7}$ in Figures 5-8. 

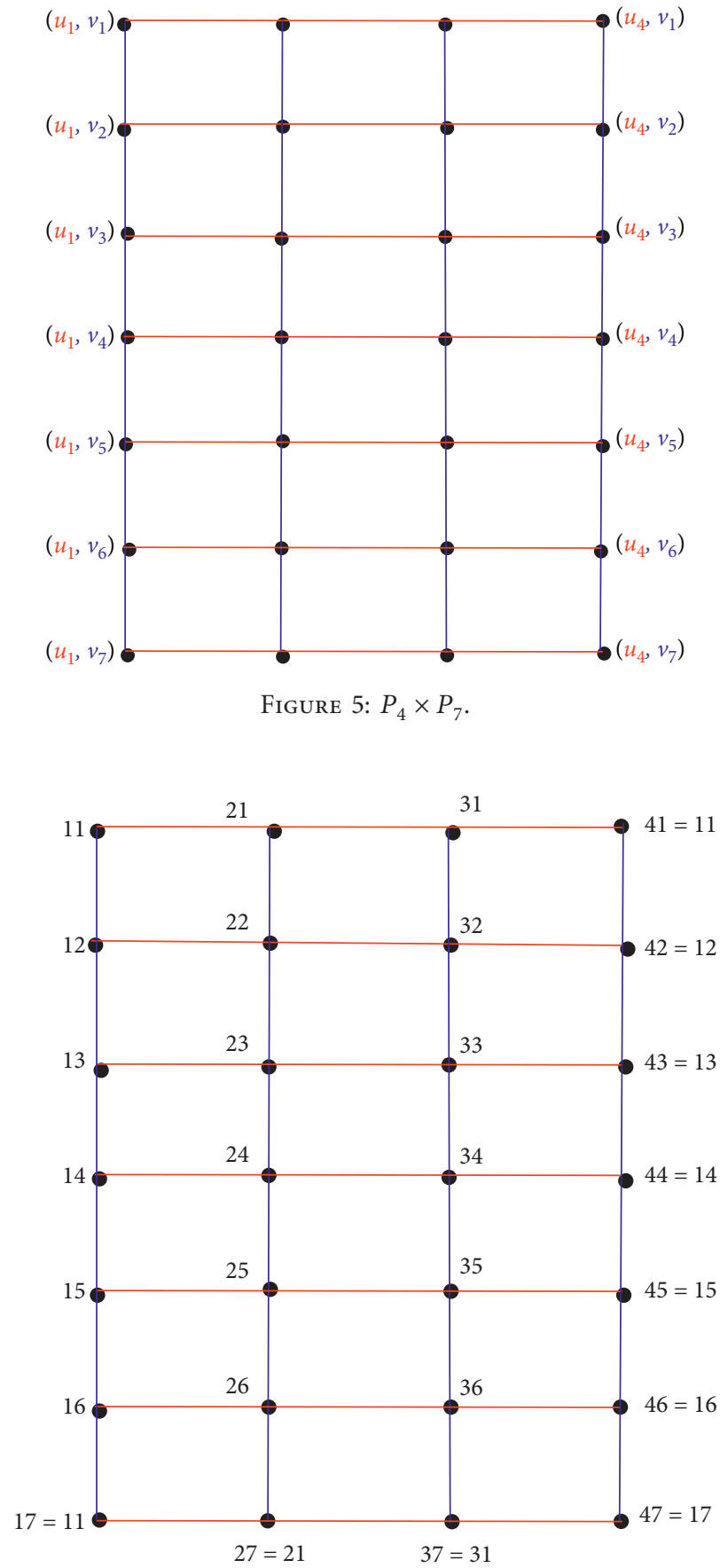

Figure 6: Grid form of $T_{4,7}$.

For brevity, we shall use the symbol $w_{i, j}\left(v_{i j}\right.$ or simply $i j$ ) to represent the vertex $\left(u_{i}, v_{j}\right)$ of $T_{m, n}$. In the following, you can see the grid form of $T_{4,7}$ with simple labels.

First of all, we identify, respectively, the vertices $v_{1, j}$ with the vertices $v_{4, j}$ and identify, respectively, edges $\left(v_{1, j}, v_{1, j+1}\right)$ with the edges $\left(v_{4, j}, v_{4, j+1}\right)$ for $1 \leq j \leq 7$ and receive a cylinder.

Finally, after identification of the vertices $v_{i, 1}$ with the vertices $v_{i, 7}$ and the edges $\left(v_{i, 1}, v_{i+1,1}\right)$ with the edges $\left(v_{i, 7}, v_{i+1,7}\right)$ for $1 \leq i \leq 3$ we get the polygonal torus $T_{4,7}$.

In the following, $m, n \geq 4$.
Theorem 3. Let $m$ and $n$ be odd. Then,

(1) $W\left(C_{m, n}\right)=(1 / 8)(m-1)(n-1)^{2}\left(m^{2}+m n+5 n-n\right.$ $+2)$

(2) $W W\left(C_{m, n}\right)=(1 / 48)(m-1)(n-1)^{2}\left(2 m^{3}+2 m^{2} n+\right.$ $\left.2 m n^{2}+21 m^{2}-4 m n-2 n^{2}+52 m+n+21\right)$

Proof. We prove it using the distance matrix $D$ corresponding to the polygonal torus $T_{m, n}$, which is symmetric and have order $(m-1)(n-1) \times(m-1)(n-1)$. Each row of $D$ represents the distances from a vertex $v_{i j}$ to the vertices 


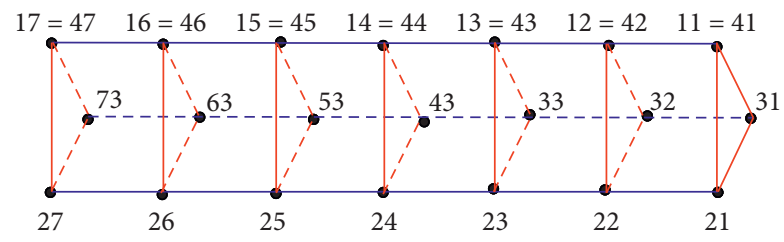

Figure 7: First fold of $P_{4} \times P_{7}$.

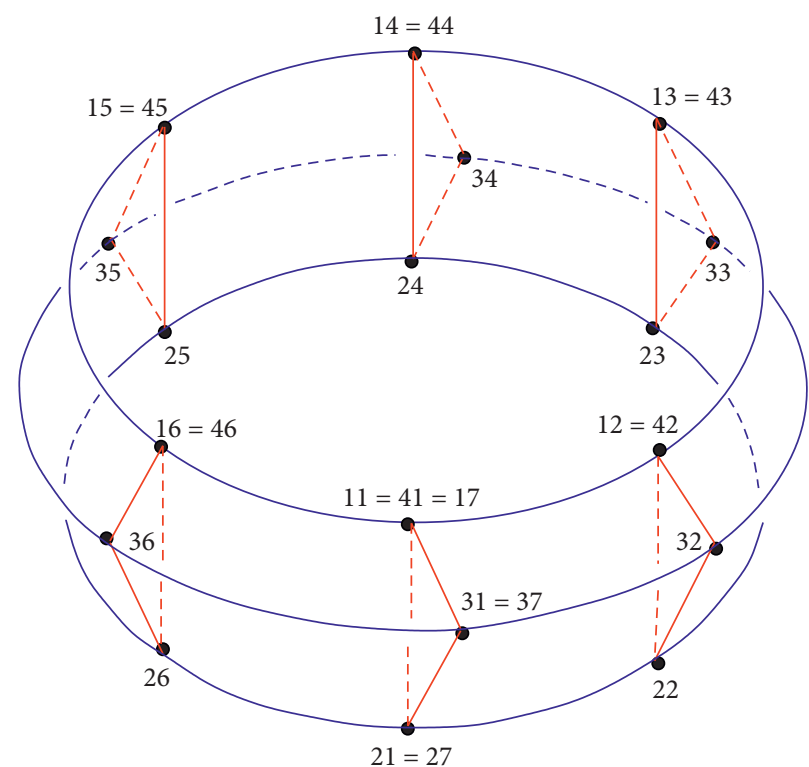

Figure 8: $T_{4,7}$.

$v_{1,1}, v_{1,2}, \ldots, v_{1, n}, v_{2,1}, v_{2,2}, \ldots, v_{2, n}, \ldots, v_{m-1,1}, v_{m-1,2}, \ldots, v_{m}$ $-1, n$, respectively. Since we need distinct paths, we shall consider only its upper-triangular part. For this, we represent the upper-triangular part by submatrices. There are $(m-1 / 2)$ distinct submatrices $A_{0}, A_{1}, A_{2}, A_{3}, \ldots$, $A_{(m-3 / 2)}, A_{(m-1 / 2)}$. All these submatrices are symmetric, each having order $(n-1) \times(n-1)$. Each $A_{i}$ appears $m-1$ times except $A_{(m-1 / 2)}$, which appears $(m-1 / 2)$ times. $A_{0}$ appears only on the main diagonal of $D . A_{i}, 1 \leq i \leq(m-3 / 2)$, appears $m-(i+1)$ times in $i$ th secondary diagonal and $i$ times in $(m-(i-1))$ th secondary diagonal. $A_{(m-1 / 2)}$ appears only in $(m-1 / 2)$ th secondary diagonal.

The rows of $D$ are

$$
\begin{aligned}
R_{1} & =\left(A_{0}, A_{1}, A_{2}, \ldots, A_{(m-3 / 2)}, A_{(m-1 / 2)}, A_{(m-3 / 2)}, A_{(m-5 / 2)}, \ldots, A_{2}, A_{1}\right), \\
R_{2} & =\left(A_{1}, A_{0}, A_{1}, \ldots, A_{(m-5 / 2)}, A_{(m-3 / 2)}, A_{(m-1 / 2)}, A_{(m-3 / 2)}, \ldots, A_{3}, A_{2}\right), \\
R_{3} & =\left(A_{2}, A_{1}, A_{0}, \ldots, A_{(m-7 / 2)}, A_{(m-5 / 2)}, A_{(m-3 / 2)}, A_{(m-1 / 2)}, \ldots, A_{4}, A_{3}\right), \\
R_{4} & =\left(A_{3}, A_{2}, A_{1}, \ldots, A_{(m-7 / 2)}, A_{(m-5 / 2)}, A_{(m-3 / 2)}, A_{(m-1 / 2)}, \ldots A_{5}, A_{4}\right), \\
& \vdots \\
R_{(m-1)(n-1)} & =\left(A_{1}, A_{2}, A_{3}, \ldots, A_{(m-1 / 2)}, A_{(m-3 / 2)}, \ldots, A_{1}, A_{0}\right) .
\end{aligned}
$$


The rows of $A_{i}, 0 \leq i \leq(m-1 / 2)$, are

$$
\begin{aligned}
& R_{1}=\left(i, i+1, i+2, i+3, \ldots, i+\frac{n-1}{2}-1, i+\frac{n-1}{2}, i+\frac{n-1}{2}-1, i+\frac{n-1}{2}-2, \ldots, i+2, i+1\right), \\
& R_{2}=\left(i+1, i, i+1, i+2, \ldots, i+\frac{n-1}{2}-2, i+\frac{n-1}{2}-1, i+\frac{n-1}{2}, i+\frac{n-1}{2}-1, \ldots, i+3, i+2\right), \\
& R_{3}=\left(i+2, i+1, i, i+1, \ldots, i+\frac{n-1}{2}-3, i+\frac{n-1}{2}-2, i+\frac{n-1}{2}-1, i+\frac{n-1}{2}, \ldots, i+4, i+3\right), \\
& \vdots \\
& R_{(n-1 / 2)}=\left(i+\frac{n-1}{2}-1, i+\frac{n-1}{2}-2, i+\frac{n-1}{2}-3, \ldots, i+1, i, i+1, i+2, \ldots, i+\frac{n-1}{2}-2, i+\frac{n-1}{2}-1, i+\frac{n-1}{2}\right), \\
& R_{(n-1 / 2)+1}=\left(i+\frac{n-1}{2}, i+\frac{n-1}{2}-1, i+\frac{n-1}{2}-2, \ldots, i+2, i+1, i, i+1, \ldots, i+\frac{n-1}{2}-3, i+\frac{n-1}{2}-2, i+\frac{n-1}{2}-1\right), \\
& R_{(n-1 / 2)+2}=\left(i+\frac{n-1}{2}-1, i+\frac{n-1}{2}, i+\frac{n-1}{2}-1, \ldots, i+3, i+2, i+1, i, \ldots, i+\frac{n-1}{2}-4, i+\frac{n-1}{2}-3, i+\frac{n-1}{2}-2\right), \\
& \vdots \\
& R_{n-2}=\left(i+2, i+3, i+4, \ldots, i+\frac{n-1}{2}-1, i+\frac{n-1}{2}-2, i+\frac{n-1}{2}-3, \ldots, i+1, i, i+1\right), \\
& R_{n-1}=\left(i+1, i+2, i+3, \ldots, i+\frac{n-1}{2}, i+\frac{n-1}{2}-1, i+\frac{n-1}{2}-2, \ldots, i+2, i+1, i\right) .
\end{aligned}
$$

Here, all entries of each $A_{i}$ contribute towards counting except $A_{0}$, whose only upper-triangular entries contribute. The remaining part of the proof is similar to the proof of Theorem 1.

Theorem 4. Let $m$ be odd and $n$ be even. Then,

(1) $W\left(C_{m, n}\right)=(1 / 8)(m-1)(n-1)\left(m^{2} n+m n^{2}-m^{2}+\right.$ $\left.4 m n-n^{2}-6 m+3 n-1\right)$
(2) $W W\left(C_{m, n}\right)=(1 / 48)(m-1)(n-1) \quad\left(2 m^{3} n+\right.$ $3 m^{2} n^{2}+2 m n^{3}-2 m^{3}+18 m^{2} n-4 m n^{2}-2 n^{3}-24 m^{2}$ $\left.+50 m n+3 n^{2}-46 m+26 n-24\right)$

Proof. $D$ is the same as in Theorem 3. However, the entries of $A_{i}, 0 \leq i \leq(m-1 / 2)$, are different and are 


$$
\begin{aligned}
& R_{1}=\left(i, i+1, i+2, i+3, \ldots, i+\frac{n}{2}-2, i+\frac{n}{2}-1, i+\frac{n}{2}-1, i+\frac{n}{2}-2, i+\frac{n}{2}-3, \ldots, i+2, i+1\right), \\
& R_{2}=\left(i+1, i, i+1, i+2, \ldots, i+\frac{n}{2}-3, i+\frac{n}{2}-2, i+\frac{n}{2}-1, i+\frac{n}{2}-1, i+\frac{n}{2}-2, \ldots, i+3, i+2\right), \\
& R_{3}=\left(i+2, i+1, i, i+1, \ldots, i+\frac{n}{2}-4, i+\frac{n}{2}-3, i+\frac{n}{2}-2, i+\frac{n}{2}-1, i+\frac{n}{2}-1, \ldots, i+4, i+3\right), \\
& \vdots \\
& R_{(n / 2)-1}=\left(i+\frac{n}{2}-2, i+\frac{n}{2}-3, i+\frac{n}{2}-4, \ldots, i+1, i, i+1, i+2, \ldots, i+\frac{n}{2}-2, i+\frac{n}{2}-1, i+\frac{n}{2}-1\right) \\
& R_{(n / 2)}=\left(i+\frac{n}{2}-1, i+\frac{n}{2}-2, i+\frac{n}{2}-3, \ldots, i+2, i+1, i, i+1, \ldots, i+\frac{n}{2}-3, i+\frac{n}{2}-2, i+\frac{n}{2}-1\right) \\
& R_{(n / 2)+1}=\left(i+\frac{n}{2}-1, i+\frac{n}{2}-1, i+\frac{n}{2}-2, \ldots, i+3, i+2, i+1, i, \ldots, i+\frac{n}{2}-4, i+\frac{n}{2}-3, i+\frac{n}{2}-2\right), \\
& R_{(n / 2)+2}=\left(i+\frac{n}{2}-2, i+\frac{n}{2}-1, i+\frac{n}{2}-1, \ldots, i+4, i+3, i+2, i+1, \ldots, i+\frac{n}{2}-5, i+\frac{n}{2}-4, i+\frac{n}{2}-3\right) \\
& \vdots \\
& R_{n-2}=\left(i+2, i+3, i+4, \ldots, i+\frac{n}{2}-1, i+\frac{n}{2}-1, i+\frac{n}{2}-2, i+\frac{n}{2}-3, \ldots, i+1, i, i+1\right), \\
& R_{n-1}=\left(i+1, i+2, i+3, \ldots, i+\frac{n}{2}-2, i+\frac{n}{2}-1, i+\frac{n}{2}-1, i+\frac{n}{2}-2, i+\frac{n}{2}-3, \ldots, i+2, i+1, i\right) .
\end{aligned}
$$

Theorem 5. Let $m$ be even and $n$ be odd. Then,

(1) $W\left(C_{m, n}\right)=(1 / 8)(m-1)(n-1)^{2}\left(m^{2}+m n+5 m-\right.$ $n+9)$

(2) $W W\left(C_{m, n}\right)=(1 / 48)(m-1)(n-1)^{2}\left(2 m^{3}+3 m^{2} n+\right.$ $2 m n^{2}+21 m^{2}-4 m n-2 n^{2}+94 m-2 n+96$
Proof. Here, $D$ has order $(m-1)(n-1) \times(m-1)(n-1)$, and there are $(m / 2)-1$ distinct submatrices $A_{0}, A_{1}, A_{2}, A_{3}, \ldots, A_{(m / 2)-2}$ and $A_{(m / 2)-1}$. All these submatrices are symmetric, each having order $(n-1) \times(n-1)$. Each $A_{i}$ appears $m-1$ times. $A_{0}$ appears only on the main diagonal of $D$. $A_{i}, 1 \leq i \leq(m / 2)-1$, appears $m-(i+1)$ times in $i$ th secondary diagonal and $i$ times in $[m-(i-$ 1)] th secondary diagonal. The rows of $D$ are

$$
\begin{aligned}
R_{1} & =A_{0}, A_{1}, A_{2}, \ldots, A_{(m / 2)-2}, A_{(m / 2)-1}, A_{(m / 2)-1}, A_{(m / 2)-2}, A_{(m / 2)-3}, \ldots, A_{2}, A_{1}, \\
R_{2} & =A_{1}, A_{0}, A_{1}, \ldots, A_{(m / 2)-3}, A_{(m / 2)-2}, A_{(m / 2)-1}, A_{(m / 2)-1}, A_{(m / 2)-2}, \ldots, A_{3}, A_{2}, \\
R_{3} & =A_{2}, A_{1}, A_{0}, \ldots, A_{(m / 2)-3}, A_{(m / 2)-2}, A_{(m / 2)-1}, A_{(m / 2)-1}, A_{(m / 2)-2}, \ldots, A_{4}, A_{3}, \\
& \vdots \\
R_{(m-1)(n-1)} & =A_{1}, A_{2}, A_{3}, \ldots, A_{(m / 2)-1}, A_{(m / 2)-1}, A_{(m / 2)-2}, \ldots, A_{2}, A_{1}, A_{0} .
\end{aligned}
$$

The submatrices $A_{i}, 0 \leq i \leq(m / 2)-1$, are the same as thosein Theorem 3. The proofs of $c_{k}, c_{(n-1 / 2)}$, and $c_{(n-1 / 2)+k}$ are already given in Theorem 3. We need only $c_{(m / 2)+k}, 0 \leq k \leq(n-1 / 2)$ : 
TABLe 1: Wiener and hyper-Wiener indices the cylinder.

\begin{tabular}{lcccc}
\hline Result & $n$ & $m$ & $W$ & WW \\
\hline Theorem 1 & 5 & 5 & 520 & 1130 \\
Theorem 1 & 6 & 9 & 4544 & 32280 \\
Theorem 2 & 3 & 8 & 574 & 1211 \\
Theorem 2 & 4 & 12 & 3850 & 11275 \\
\hline
\end{tabular}

TABle 2: Wiener and hyper-Wiener indices of the torus.

\begin{tabular}{lcccc}
\hline Result & $n$ & $m$ & $W$ & WW \\
\hline Theorem 3 & 5 & 13 & 7104 & 46080 \\
Theorem 3 & 7 & 21 & 61920 & 2415 \\
Theorem 4 & 4 & 11 & 23440 & 1393040 \\
Theorem 4 & 6 & 17 & 103360 & 187360 \\
Theorem 5 & 9 & 20 & 275425 & 1001072 \\
Theorem 5 & 11 & 24 & 42126 & 3142950 \\
Theorem 6 & 8 & 18 & 133245 & 343196 \\
Theorem 6 & 10 & 22 & 1323000 \\
\hline
\end{tabular}

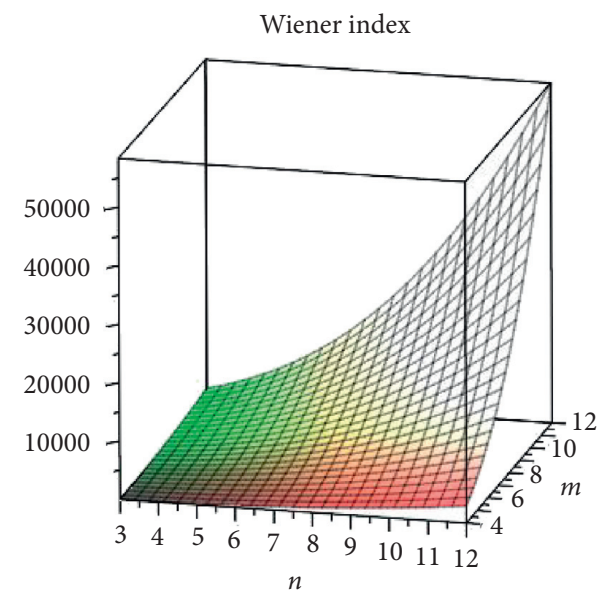

(a)

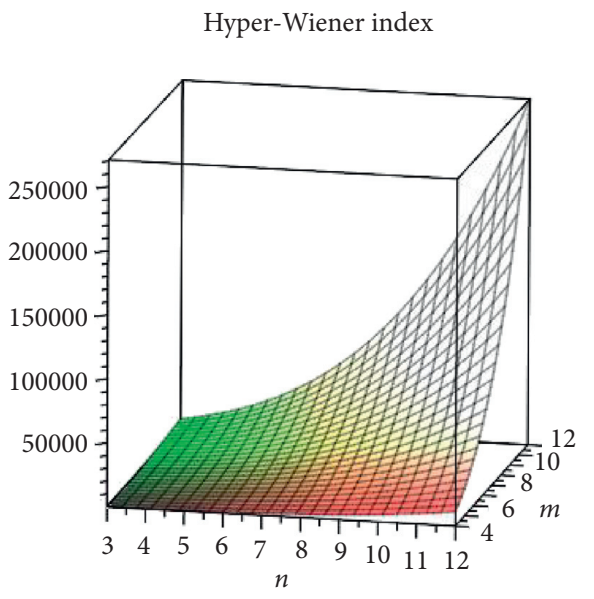

(b)

FIgURE 9: (a) Wiener index and (b) hyper-Wiener index for even $m$.

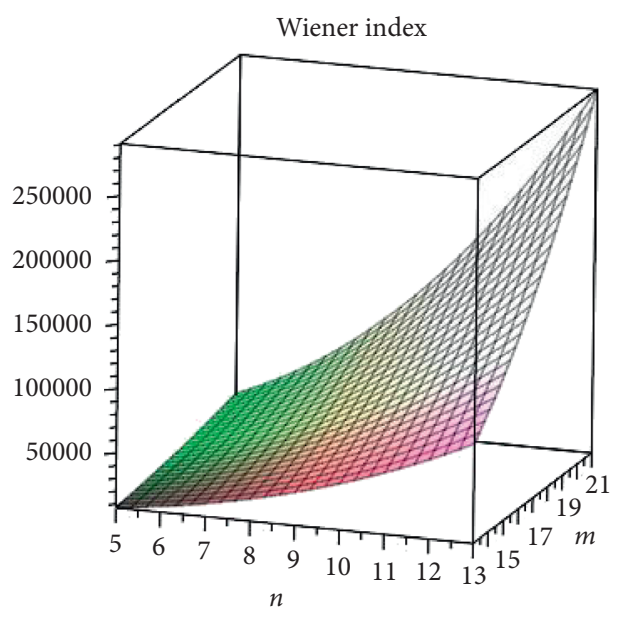

(a)

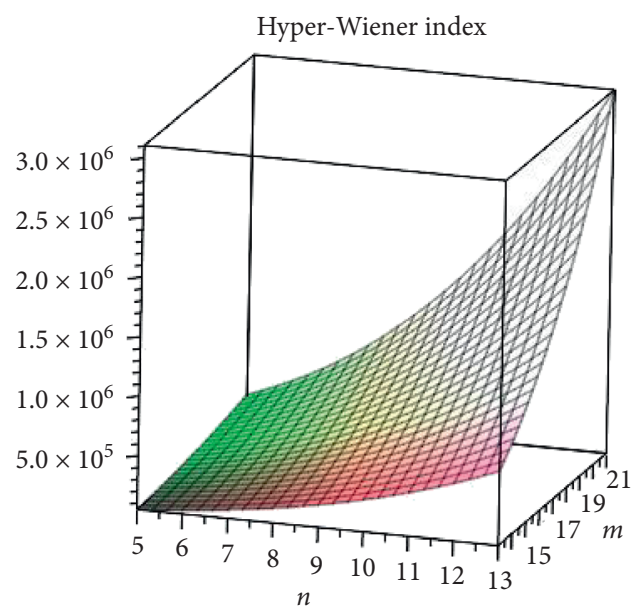

(b)

Figure 10: (a) Wiener index and (b) hyper-Wiener index for odd $m$ and $n$. 


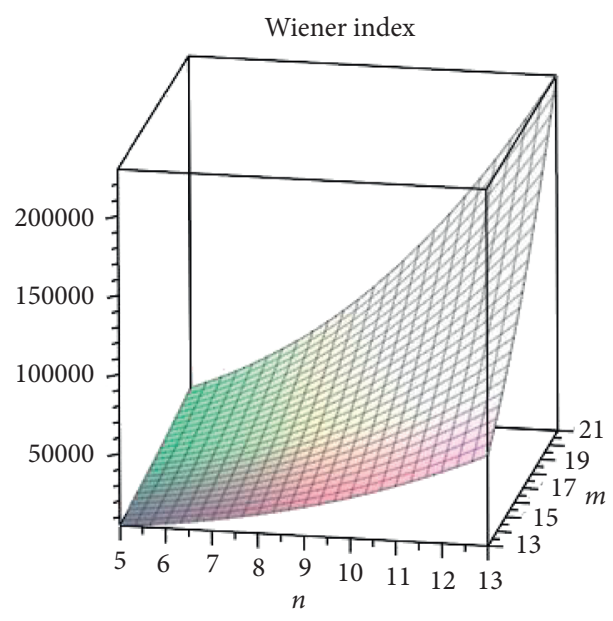

(a)

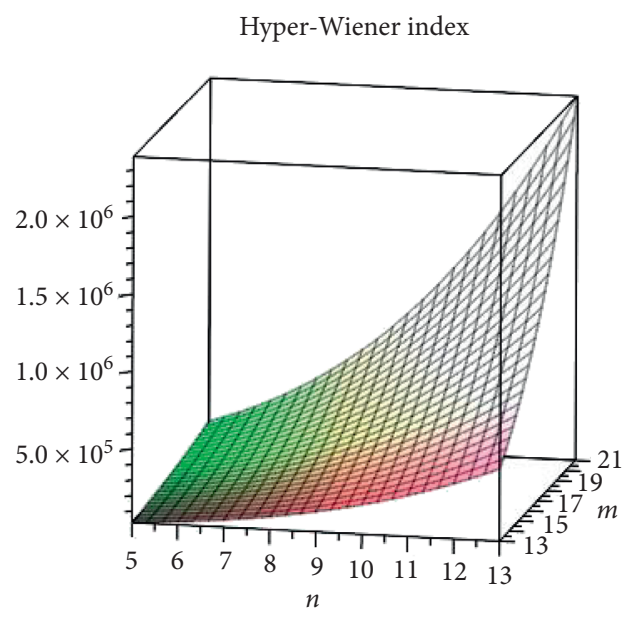

(b)

Figure 11: (a) Wiener index and (b) hyper-Wiener index for even $m$ and $n$.

$$
\begin{aligned}
\mathcal{c}_{(m / 2)+k}= & \left.\#\left(\frac{m}{2}+k\right) \in A_{(m / 2)-(n-1 / 2)+k}\right] \times\left[\#\left(A_{(m / 2)-(n-1 / 2)+k}\right)\right] \\
& +\left[\#\left(\frac{m}{2}+k\right) \in A_{(m / 2)-(n-1 / 2)+k+1}\right] \times\left[\#\left(A_{(m / 2)-(n-1 / 2)+k+1}\right)\right]+\left[\#\left(\frac{m}{2}+k\right) \in A_{(m / 2)-(n-1 / 2)+k+2}\right] \\
& \times\left[\#\left(A_{(m / 2)-(n-1 / 2)+k+2}\right)\right]+\cdots+\left[\#\left(\frac{m}{2}+k\right) \in A_{(m / 2)-2}\right] \times\left[\#\left(A_{(m / 2)-2}\right)\right] \\
& +\left[\#\left(\frac{m}{2}+k\right) \in A_{(m / 2)-1}\right] \times\left[\#\left(A_{(m / 2)-1}\right)\right] \\
= & (n-1)(m-1)+2(n-1)(m-1)+2(n-1)(m-1)+\cdots+2(n-1)(m-1)+2(n-1)(m-1) \\
= & (n-1)(m-1)(1+2+2+\cdots+2+2) \\
= & (n-1)(m-1)[n-2(k+1)] .
\end{aligned}
$$

Theorem 6. For even $m$ and $n$, we obtain

(1) $W\left(C_{m, n}\right)=(1 / 8)(m-1)(n-1)(m+n-2)(m n-$ $m-n)$

(2) $W W\left(C_{m, n}\right)=(1 / 48)(m-1)(n-1)\left(2 m^{3} n+\right.$ $2 m^{2} n^{2}+2 m n^{3}-2 m^{3}-6 m^{2} n-6 m n^{2}-2 n^{3}-4 m n+$ $8 m+n)$
Proof. Here, the distance matrix $D$ is the same as that in Theorem 5. Also, the submatrices $A_{i}, 0 \leq i \leq(m / 2)-1$ are the same as those in Theorem 4 . The proofs of $c_{k}$ and $c_{(n / 2)+k}$ are similar to proofs given in Theorem 4 . We need only $c_{(m / 2)+k}, 0 \leq k \leq(n / 2)-2$ :

$$
\begin{aligned}
\mathcal{c}_{(m / 2)+k}= & \left.\#\left(\frac{m}{2}+k\right) \in A_{(m / 2)-(n / 2)+k+1}\right] \times\left[\#\left(A_{(m / 2)-(n / 2)+k+1}\right)\right]+\left[\#\left(\frac{m}{2}+k\right) \in A_{(m / 2)-(n / 2)+k+2}\right] \\
& \times\left[\#\left(A_{(m / 2)-(n / 2)+k+2}\right)\right]+\left[\#\left(\frac{m}{2}+k\right) \in A_{(m / 2)-(n / 2)+k+3}\right] \times\left[\#\left(A_{(m / 2)-(n / 2)+k+3}\right)\right]+\cdots+\left[\#\left(\frac{m}{2}+k\right) \in A_{(m / 2)-2}\right] \\
& \times\left[\#\left(A_{(m / 2)-2}\right)\right]+\left[\#\left(\frac{m}{2}+k\right) \in A_{(m / 2)-1}\right] \times\left[\#\left(A_{(m / 2)-1}\right)\right] \\
= & 2(n-1)(m-1)+2(n-1)(m-1) \\
& +2(n-1)(m-1)+\cdots+2(n-1)(m-1)+2(n-1)(m-1) \\
= & (n-1)(m-1)(2+2+2+\cdots+2+2) \\
= & (n-1)(m-1)[n-2(k+1)] .
\end{aligned}
$$




\section{Conclusion and Discussion}

In this paper, we introduced two objects, polygonal cylinder and polygonal torus, using Cartesian product of paths $P_{m}$ and $P_{n}$ and using topological identifications. We hope that it will play a useful role in studying molecular graphs, particularly, nanotubes and nanocones, involving them as subgraphs. Secondly, we gave the general closed forms their Wiener and hyper-Wiener indices. We found all possible cases of the indices depending on odd and even $m$ and $n$. The approach to compute these indices was simple, quick, and interesting.

Actually, we first enlisted all the distances as entries of a matrix and then found its submatrices to count the paths. You can see the values of these indices for several different values of $m$ and $n$ in Tables 1 and 2 and can see the graphs of Wiener and hyper-Wiener indices in Figures 9-11. To simplify long expressions we used MAPLE.

\section{Data Availability}

No data are included in this article.

\section{Disclosure}

The preprint of this paper is available on the Research Gate (researchgate.net/publication/335651873).

\section{Conflicts of Interest}

Authors declare that there are no conflicts of interest.

\section{Acknowledgments}

This work was supported by the Humanities and Social Sciences Project of Anhui Provincial Education Department "Research on social crisis management in Anhui cities under the background of big data" (subject no. SK2016A0233).

\section{References}

[1] H. Wiener, "Structural determination of paraffin boiling points," Journal of the American Chemical Society, vol. 69, no. 1, pp. 17-20, 1947.

[2] H. Wiener, "Correlation of heats of isomerization, and differences in heats of vaporization of isomers, among the paraffin hydrocarbons," Journal of the American Chemical Society, vol. 69, no. 11, pp. 2636-2638, 1947.

[3] H. Wiener, "Influence of interatomic forces on paraffin properties," The Journal of Chemical Physics, vol. 15, no. 10, p. 766, 1947.

[4] H. Wiener, "Vapor pressure-temperature relationships among the branched paraffin hydrocarbons," The Journal of Physical and Colloid Chemistry, vol. 52, no. 2, pp. 425-430, 1948.

[5] H. Wiener, "Relation of the physical properties of the isomeric alkanes to molecular structure. Surface tension, specific dispersion, and critical solution temperature in aniline," The Journal of Physical and Colloid Chemistry, vol. 52, no. 6, pp. 1082-1089, 1948.
[6] I. Gutman and T. Kortevlyesi, "Wiener Indices and Molecular Surfaces, Zeitschrift Fur Naturforschung - Section," A Journal of Physical Sciences, vol. 50, pp. 669-671, 1995.

[7] M. V. Diudea and O. Ivanciuc, Molecular Topology, Comprex, Cluj, Romania, 1995.

[8] I. Gutman and J. H. Potgieter, "Wiener index and intermolecular forces," South African Journal of Science, vol. 92, pp. 47-48, 1996.

[9] I. Gutman and J. H. Potgieter, "Wiener index and intermolecular forces," Journal of Serbian Chemical Society, vol. 62, pp. 185-192, 1997.

[10] S. G. Wagner, "A class of trees and its wiener index," Acta Applicandae Mathematicae, vol. 91, no. 2, pp. 119-132, 2006.

[11] H. Wang and G. Yu, "All but 49 numbers are wiener indices of trees," Acta Applicandae Mathematicae, vol. 92, no. 1, pp. 15-20, 2006.

[12] H. Hua and M. Wang, "On harary index and traceable graphs," MATCH Communications in Mathematical and in Computer Chemistry, vol. 70, pp. 297-300, 2013.

[13] H. Hua and B. Ning, "Wiener index, harary index and hamiltonicity of graphs," MATCH Communications in Mathematical and in Computer Chemistry, vol. 78, pp. 153$162,2016$.

[14] R. Khalid and N. Idrees, "Wiener index and hyper-wiener index of Dutch windmill graph," UPB Scientific Bulletin, Series $B$, vol. 80, pp. 31-38, 2018.

[15] C. Thilaga and P. B. Sarasija, "Wiener and hyper-wiener indices of unitary addition Cayley graphs," International Journal of Recent Technology and Engineering (IJRTE), vol. 8, pp. 131-132, 2019.

[16] I. Gutman, Y. N. Yeh, S. L. Lee, and Y. L. Luo, "Some recent results in the theory of the wiener number," Indian Journal of Chemistry, vol. 32, pp. 651-661, 1993.

[17] N. Bosnjak, Z. Mihali, and N. Trinajsti, Journal of Chromatography, vol. 540, pp. 430-434, 1991.

[18] I. Lukovits, "Decomposition of the wiener topological index. Application to drug-receptor interactions," Journal of the Chemical Society, Perkin Transactions, vol. 2, no. 9, pp. 1667-1671, 1988.

[19] D. H. Rouvray, "The modeling of chemical phenomena using topological indices," Journal of Computational Chemistry, vol. 8 , no. 4, pp. 470-480, 1987.

[20] I. n. Lukovits, "Correlation between components of the Wiener index and partition coefficients of hydrocarbons," International Journal of Quantum Chemistry, vol. 44, no. S19, pp. 217-223, 1992.

[21] S. Nikolic, N. Trinajstic, and Z. Mihalic, "On mathematical properties of buckminsterfullerene," Croatica Chemica Acta, vol. 68, pp. 105-129, 1995.

[22] D. H. Rouvray, Chemical Applications of Topology and Graph Theory, pp. 159-177, Elsevier, Amsterdam, The Netherland, 1983.

[23] D. H. Rouvray and N. Trinajstic, Mathematics and Computational Concepts in Chemistry, pp. 295-306, Horwood, Chichester, UK, 1986.

[24] D. Bonchev, Information Theoretic Indices for Characterization of Chemical Structure, Research Studies Press, Chichester, UK, 1983.

[25] D. Bonchev and D. H. Rouvray, Chemical Graph TheoryIntroduction and Fundamentals, Gordon and Breach, New York, NY, USA, 1991.

[26] M. V. Diudea, "Wiener and hyper-wiener numbers in a single matrix," Journal of Chemical Information and Computer Sciences, vol. 36, no. 4, pp. 833-836, 1996. 
[27] M. R. Farahani, "On the schultz polynomial and Hosoya polynomial of circumcoronene series of benzenoid," Journal of Applied Mathematics \& Informatics, vol. 31, no. 5_6, pp. 595-608, 2013.

[28] A. Graovac, I. Gutman, and N. Trinajsti, Topological Approach to the Chemistry of Conjugated Molecules, Springer-Verlag, Berlin, Germany, 1977.

[29] I. Gutman, Some Properties of the Wiener Polynomials, vol. 25, pp. 13-18, Graph Theory, New York, NY, USA, 1993.

[30] I. Gutman and O. E. Polansky, Mathematical Concepts in Organic Chemistry, Springer-Verlag, Berlin, Germany, 1986.

[31] I. Gutman, W. Linert, I. Lukovits, and A. A. Dobrynin, "Trees with Extremal Hyper-Wiener index: mathematical basis and chemical applications," Journal of Chemical Information and Computer Sciences, vol. 37, no. 2, pp. 349-354, 1997.

[32] P. J. Hansen and P. C. Jurs, "Chemical applications of graph theory. Part I. Fundamentals and topological indices," Journal of Chemical Education, vol. 65, no. 7, pp. 574-580, 1988.

[33] S. Hayat, M. A. Malik, and M. Imran, "Computing topological indices of honey-comb derived networks," Romanian Journal of Information Science and Technology, vol. 18, pp. 144-165, 2015.

[34] M. Randic, "Novel molecular descriptor for structure-property studies," Chemical Physics Letters, vol. 211, pp. 478-483, 1993.

[35] G. Cash, S. Petkovšek, and M. Petkovsek, "Three methods for calculation of the hyper-wiener index of molecular graphs," Journal of Chemical Information and Computer Sciences, vol. 42, no. 3, pp. 571-576, 2002.

[36] M. H. Khalifeh, H. Yousefi-Azari, and A. R. Ashrafi, "The hyper-Wiener index of graph operations," Computers and Mathematics with Applications, vol. 56, no. 5, pp. 1402-1407, 2008.

[37] D. J. Klein, I. Lukovits, and I. Gutman, "On the definition of the hyper-wiener index for cycle-containing structures," Journal of Chemical Information and Modeling, vol. 35, no. 1, pp. 50-52, 1995.

[38] I. Lukovits and W. Linert, "A novel definition of the hyperwiener index for cycles," Journal of Chemical Information and Modeling, vol. 34, no. 4, pp. 899-902, 1994.

[39] M. Darafsheh, M. Khalifeh, and H. Jolany, "The hyper-wiener index of one-pentagonal carbon nanocone," Current Nanoscience, vol. 9, no. 4, pp. 557-560, 2013.

[40] M. Randic, X. Guo, T. Oxley, and H. Krishnapriyan, "Wiener matrix: source of novel graph invariants," Journal of Chemical Information and Computer Sciences, vol. 33, no. 5, pp. 709716, 1993.

[41] P. N. Kishori and S. B. Lokesh, "Veena mathad and ivan gutman, "hosoay polynomial of hanoi graphs," Kragujevac Journal of Mathematics, vol. 36, pp. 51-57, 2012. 